\title{
Exogenous Ketone Supplement Ingestion and Submaximal Exercise Response in Trained, Male Cyclists: A Randomized, Double-blind Study
}

\author{
${ }^{1}$ Devon Da Costa, ${ }^{2}$ Varsha Banagalee, ${ }^{1}$ Rowena Naidoo* \\ ${ }^{1}$ College of Health Sciences, Discipline of Biokinetics, Exercise and Leisure Sciences, University of \\ KwaZulu-Natal, South Africa. ${ }^{2}$ College of Health Sciences, Discipline of Pharmaceutical Sciences, \\ University of KwaZulu-Natal, South Africa.
}

Submitted 06 June 2020; Accepted in final form 01 August 2020.

\begin{abstract}
Background. The acute ingestion of ketone body supplements can improve exercise responses such as oxygen consumption and blood lactate. Additionally, ketone body supplements show increases in circulating $\beta \mathrm{hb}$ concentration, matching those of someone in a state of nutritional ketosis. Studies testing ketone body supplements have reported different results in exercise response and varying gastrointestinal discomfort. Objectives. This study aimed to compare the effects of exogenous ketone supplements on submaximal exercise response in a group of trained male cyclists. Methods. A randomized, double-blind study was conducted. Forty-four males were randomly assigned to one of four supplement groups, i.e., high ketone (HKET) (10g $\beta \mathrm{hb})$; low ketone (LKET) ( $4 \mathrm{~g} \beta \mathrm{hb}$ ); carbohydrate (CHO); or placebo (PLA). Oxygen consumption and blood lactate were measured as markers of exercise response and blood $\beta \mathrm{hb}$ as a marker for ketosis, during a submaximal cycle protocol (30 min at 60\% VO2max). Results. Two of the three supplements increased oxygen consumption (CHO: $\mathrm{p}<0.0005$; and HKET: $\mathrm{p}=0.015$ ). The HKET group experienced an increase in maximal blood lactate $(\mathrm{p}=0.010)$ and a decrease in lactate threshold $(\mathrm{d}=0.93)$. Additionally, the HKET group showed the largest increase in circulating $\beta \mathrm{hb}$ concentration $(\mathrm{p}=0.004) 30$ minutes following ingestion. The respiratory exchange ratio, heart rate, and plasma glucose were not affected. Conclusion. Although the exogenous ketone supplements were able to induce a mild state of ketosis $(>0.5 \mathrm{mmol} / \mathrm{L})$, no positive effect on exercise response was shown.
\end{abstract}

KEYWORDS: Beta-Hydroxybutyrate, Ketone Bodies, Ketone Supplements, Cycling Exercise Performance.

\section{INTRODUCTION}

Nutritional strategies and supplements are used to enhance exercise performance for recreational sports-persons and elite athletes. These can also be used to achieve the nutritional state of ketosis: when circulating $\beta \mathrm{hb}$ is above > $0.5 \mathrm{mmol} / \mathrm{L}(1-3)$. In this state, the body no longer relies on glucose metabolization, but rather the metabolization of ketone bodies as fuel for the brain, heart, and skeletal muscles $(1,2,4)$. Several different methods are used to achieve ketosis. Amongst these are starvation, exercising, and nutrient manipulation. Alternatively, ingesting exogenous ketone body supplements containing either ketone salts or ketone esters $(1,2,5-7)$. There are three kinds of ketone bodies: acetone, acetoacetic acid, and beta-hydroxybutyrate $(\beta \mathrm{hb})$. Commercial ketone body supplements contain 8$12 \mathrm{~g} \beta \mathrm{hb}$, combined with potassium, calcium, or

*. Corresponding Author:

Rowena Naidoo, Professor

E-mail: naidoor3@ukzn.ac.za 
sodium (Na- $\beta \mathrm{hb})$ - known as ketone salts (1). Ketone esters are monoesters, known as (R)-3hydroxybutyl (R)-3-hydroxybutyrate, and are salt-free. $\beta$ hb supplements can cause an increase in $\beta \mathrm{hb}$ concentration in the blood of $>0.5-<4$ $\mathrm{mmol} / \mathrm{L}, 15$ to 45 minutes after ingestion (8).

Ketone body supplements can cause enhanced exercise response in endurance athletes, including increased power output and reduced blood lactate (9). Additionally, studies report increases in lipid utilization and respiratory exchange ratio (RER) $(10,11)$. Although ketone supplements have shown positive results, negative responses such as gastrointestinal tract discomfort have occurred (2, 12-15). Fischer et al. (16) tested 86 ketone body supplements showing a mean quantity of $\sim 11.4 \pm$ $2.7 \mathrm{~g} \beta \mathrm{hb}$ present, with $50 \%$ of the supplements combining the $\beta \mathrm{hb}$ with either sodium, calcium or magnesium. The study noted the daily recommended consumption rates were exceeded, which could lead to metabolic alkalosis. Reports of immediate side effects, such as gastrointestinal tract discomfort, nausea, diarrhea, and abdominal pain, were conveyed (16).

Exogenous ketone bodies possess the ability to affect many exercise parameters, but there is little evidence-based research to support claims. This study aimed to investigate further the effects of ketone body supplements on exercise response, including cardiovascular, respiratory, metabolic responses (17). Also, it determined different quantities of $\beta \mathrm{hb}$ for inducing ketosis and tested for possible adverse side effects.

\section{MATERIALS AND METHODS}

Study Design. This randomized, double-blind study was conducted to compare a control condition (no ingestion) with four ingestion groups, one being a placebo group. Such comparisons are referred to as pre-ingestion (control) and post-ingestion.

Participants. Cyclists from a local cycling club in KwaZulu-Natal, South Africa, were recruited. Individuals who adhered to the following criteria were eligible to participate in this study: male, injury-free, and between the ages of 25 and 55 years. Individuals were excluded from the study if they were following a high-fat diet, such as the keto-diet. Fifty cyclists volunteered to participate in this study. Once the purpose and risks of the study were explained to the participants, oral and written consent was obtained.

Fifty participants completed baseline assessments. All participants were eligible to participate in the study; however, six dropped out due to scheduling problems [3], unforeseen injuries [2], and emigration [1]. The 44 participants recruited had a mean age of $42 \pm 9.4$ years and exercised $5.5 \pm 1$ day a week. The participants were randomly assigned to one of four groups: group one - high ketone (HKET); group two - low ketone (LKET); group three - carbohydrate (CHO); and group four - placebo (PLA).

Ethical Clearance. The protocol was approved by the university's Biomedical Research Ethics Committee (BFC330/18). The authors declare that all the experiments performed were per the ethical standards of the Helsinki Declaration.

Baseline Tests. Participants were advised to refrain from eating two hours before testing and avoid any unaccustomed exercise the day before. The trial was scheduled to best suit the participants, between 6:30, am, and 6:00 pm, which remained consistent throughout testing. Circulating $\beta \mathrm{hb}$ concentration was tested, and a 24-hour food log was completed to ensure the participants were not following a high-fat diet. The food $\log$ also served as a reminder to participants, who were requested to replicate their 24-hour diet before the subsequent testing session. Pre-test meals were not provided to maintain regular daily food intake. Anthropometric testing (height, weight, and bioelectrical impedance to determine body fat percentage) and a $\mathrm{VO}_{2} \max$ test were conducted to stratify the sample as per $\mathrm{VO}_{2} \max$ volumes between $40-65 \mathrm{ml} / \mathrm{kg} / \mathrm{min}$. This value was also used to set the determined workload of $60 \%$ $\mathrm{VO}_{2} \max$ for the submaximal tests during the ingestion protocol.

The $\mathrm{VO}_{2}$ max test was an incremental protocol, where participants completed a two-minute warm-up on 25 watts (W), followed by two minutes at $50 \mathrm{~W}$, with the workload increasing by $50 \mathrm{~W}$ every two minutes until the test was complete. The experiment was terminated if the participant fell below 70 revolutions per minute (RPM); if they voluntarily stopped; or upon reaching their age-predicted maximum heart rate. Blood lactate was not measured during the baseline tests as it was used only as a comparative tool between the pre- and post-ingestion tests.

Supplement Protocol. Participants were tested at two testing points: pre-ingestion and post-ingestion. (See Figure 1 for illustration of the protocol). 
Pre-Ingestion Tests. The number of days between testing was not set for each participant due to their changing work schedules. However, no participant was tested in less than 72 hours following the previous test. The participants completed a 24-hour food log, followed by blood $\beta \mathrm{hb}$ measurements. After that, pre-test measurements of blood glucose concentration, lactate, and resting heart rate were taken. The blood indicators were measured via a finger-prick blood sample using the Accutrend Plus blood test system, while the resting heart rate was measured with POLAR heart rate monitors. Following this, each participant completed a submaximal, steadystate 30-minute cycle protocol. The protocol began with a two-minute warm-up, set on $50 \mathrm{~W}$ maintaining 70 RPM. Following the warm-up, the load generator was set at a workload fixed at $60 \%$ of the maximum wattage achieved during the baseline $\mathrm{VO}_{2} \max$ test, e.g., a participant's $\mathrm{VO}_{2}$ max was achieved at $400 \mathrm{~W}$, the submaximal cycle protocol was set at $240 \mathrm{~W}$. Cadence remained at 70RPM to simulate actual cycling conditions, such as during a race where preferred rhythm ranges from 70 - 90RPM (18). Blood lactate and blood glucose concentrations were measured every five minutes via a finger-prick blood sample, along with heart rate and rate of perceived exertion (RPE). The lactate threshold was reached at the point of the test (in minutes: seconds) if blood lactate concentrations exceeded $4 \mathrm{mmol} / \mathrm{L}$. Oxygen consumption $\left(\mathrm{VO}_{2}\right)$, respiratory exchange ratio (RER), and minute ventilation (VE) were continuously measured by the computer software: Metasoft, which was synchronized to the breathing apparatus and Cortex Metalyzer by LifeMax (Johannesburg, South Africa). The test was terminated if the participant: fell below 70RPM; voluntarily stopped; or after 30 minutes. The participants performed a two-minute cool-down at 50W. Lastly, measurements of blood lactate, blood glucose, and heart rate were taken five minutes after the termination of the test.

\section{PRE-INGESTION}

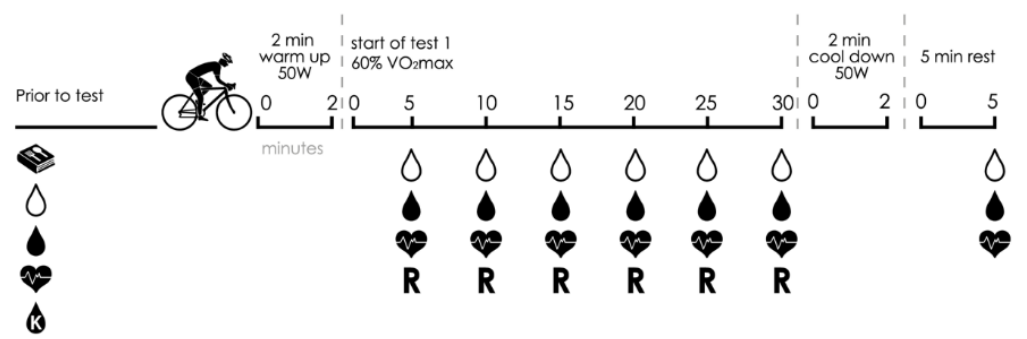

\section{POST-INGESTION}
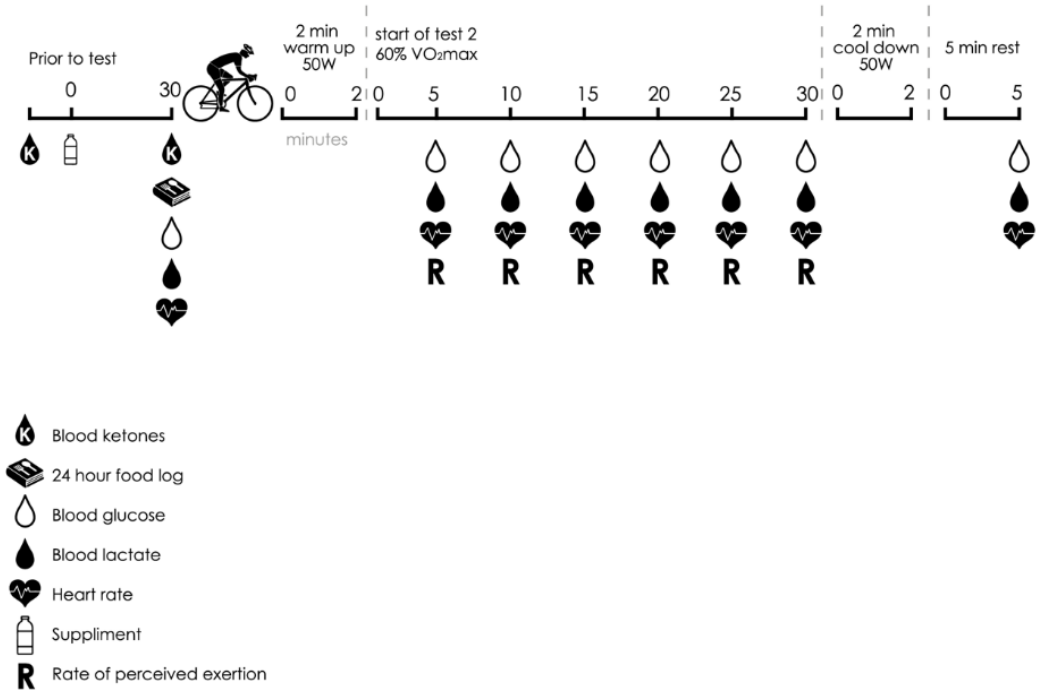

Figure 1. Following the Baseline Measurements, Participants Were Tested at Two Testing Points: Pre-Ingestion and PostIngestion. The Participants Completed the Pre-Ingestion Protocol Without any Supplements. At Least 72 Hours Following this, Participants Ingested one of the Four Supplements and Completed the Post-Ingestion Protocol. 
Post-Ingestion Tests. No less than 72 hours following the pre-ingestion tests, participants completed the testing procedure for the second time. As per pre-ingestion tests, each participant completed a 24 -hour food $\log$, and blood $\beta$ hb was measured. The supplement was ingested as per group allocation. A 30-minute waiting period was allowed to induce a state of ketosis. Studies show $\beta$ hb supplements can increase circulating blood $\beta \mathrm{hb}$ in 15 to 45 minutes $(8,9)$. Therefore, $\beta \mathrm{hb}$ concentration was re-tested 30 minutes after ingestion. Pre-tests, the cycle protocol, and posttests were followed as per the pre-ingestion process.

Supplement Content. Three supplements, plus one placebo, were manufactured to taste and look the same in a controlled laboratory by a trained pharmacist and were administered in opaque bottles. Table 1 shows the supplement group allocation and content. Supplement ingredients and $\beta \mathrm{hb}$ content were manufactured as in similar studies (2), and to imitate commercial $\beta$ hb supplements (16). The $\beta$ hb used in the study was acquired through Sigma-Aldrich and was hydroxybutyric acid, product 166898 , with a minimum purity of $95 \%$ by gas chromatography (GC).

Statistical Analysis. All data were analyzed using the Statistical Package for the Social Sciences, Version 21. Descriptive (means and standard deviations) and inferential [t-tests, analysis of covariance (ANCOVA), and repeated measures (ANOVA)] statistics were used to test significant differences pre- and postingestion. Paired t-tests were used to test for significant differences pre- to post-intervention; repeated-measures ANOVA was used to test for differences across time within groups, and ANCOVA was used to compare post-test scores between groups after correcting for pre-test scores. The changes in the means and standard deviations were compared to the pre- and postingestion scores for each group. Additionally, Cohen's d was used to describe the standardized mean difference of an effect. Effect sizes were interpreted as follows: $d>0.2$ was considered a 'small' effect size, $\mathrm{d}>0.5$ represented a 'medium' effect size and d $>0.8$ a 'large' effect size (19). Statistical significance was set at $\mathrm{p}<0.05$.

Table 1. Four Supplements Were Manufactured to Look and Taste the Same. The High Ketone Supplements Were Manufactured to Imitate Commercial $\beta$ hb Supplements (16) and Similar Studies (2). The $\beta$ hb Used Was Hydroxybutyric Acid

\begin{tabular}{|c|c|c|c|}
\hline Group & Supplement & Base Ingredients & Key Ingredients \\
\hline PLA $(\mathbf{n}=11)$ & Placebo & Citric acid: $2.32 \mathrm{~g}$; sodium bicarbonate: $2.11 \mathrm{~g}$; saccarin: $0.56 \mathrm{~g}$. & None \\
\hline CHO $(n=11)$ & Carbohydrate & Citric acid: $0.46 \mathrm{~g}$; sodium bicarbonate: $0.42 \mathrm{~g}$; saccarin: $0.5 \mathrm{~g}$. & Lactose: $4.25 \mathrm{~g}$ \\
\hline LKET $(n=11)$ & Low Ketone & Citric acid: $0.46 \mathrm{~g}$; sodium bicarbonate: $0.42 \mathrm{~g}$; saccarin: $0.5 \mathrm{~g}$. & $\beta \mathrm{hb}: 4.25 \mathrm{~g}$ \\
\hline HKET $(n=11)$ & High Ketone & Citric acid: $2.32 \mathrm{~g}$; sodium bicarbonate: $2.11 \mathrm{~g}$; saccarin: $1 \mathrm{~g}$. & $\beta \mathrm{hb}: 10 \mathrm{~g}$ \\
\hline
\end{tabular}

\section{RESULTS}

Table 2 shows the results for the baseline measurements.

Oxygen Consumption $\left(\mathbf{V O}_{2}\right)$. Oxygen consumption was affected by three of the supplements. $\mathrm{VO}_{2}$ was significantly higher postingestion than pre-ingestion for PLA $(\mathrm{p}=0.003)$, CHO $(p=0.005)$, and HKET $(p=0.015)$ groups. The LKET group showed no effect on $\mathrm{VO}_{2}$, which remained unchanged from pre- to post-ingestion. Refer to Table 3. The table shows the $\mathrm{VO}_{2}$ per supplement group measured during the pre and post-ingestion cycle protocol.

BhB Concentration. Table 4 shows circulating $\beta \mathrm{hb}$ concentration before and 30 minutes after ingestion, taken before the cycle protocol. $\beta \mathrm{hb}$ concentration was significantly higher for the HKET group 30 minutes post-ingestion. Although the HKET group presented with the largest increase $(0.089 \mathrm{mmol} / \mathrm{L}-0.367 \mathrm{mmol} / \mathrm{L}, \mathrm{p}=0.004)$, nutritional ketosis was seen only in the LKET group $(0.53 \mathrm{mmol} / \mathrm{L} \pm 0.63)$.

Table 2. Baseline Measurements

\begin{tabular}{|c|c|c|c|c|c|c|c|c|c|c|c|c|}
\hline & Age & S.D & Height & S.D & Weight & SD & B.F (\%) & S.D & HWR & S.D & BMR & SD \\
\hline Mean & 42.9 & 9.4 & 175.8 & 29.5 & 76.6 & 13.2 & 17.6 & 4.1 & 0.88 & 0.67 & 197 & 170 \\
\hline Minimum & 26 & & 185 & & 63.5 & & 8.8 & & 0.78 & & 1619 & \\
\hline Maximum & 55 & & 192 & & 90.2 & & 25.1 & & 1.06 & & 2382 & \\
\hline
\end{tabular}
SD, standard deviation; BF (5), Body fat percentage; HWR, Height-Waist Ratio; BMR, Basil Metabolic Rate. 
Table 3. Oxygen Consumption (VO2)

\begin{tabular}{lcccc}
\hline Supplementation & Mean & SD & P-Value & Cohen's D \\
\hline CHO & & & $0.005^{*}$ & 0.735 \\
$\quad$ Pre-ingestion & 43.1 & 7.1 & \\
$\quad$ Post-ingestion & 48.6 & 7.9 & $0.003^{*}$ & $1.174^{*}$ \\
PLA & 43.6 & & -0.005 \\
$\quad$ Pre-ingestion & 49.9 & 6.9 & 0.984 \\
$\quad$ Post-ingestion & 47.9 & 3.8 & \\
LKET & 47.9 & 8.6 & $0.858^{*}$ \\
$\quad$ Pre-ingestion & & 5.1 & \\
$\quad$ Post-ingestion & 40.5 & 5.5 & & \\
HKET & 44.9 & 4.7 & & \\
$\quad$ Pre-ingestion & & & \\
$\quad$ Post-ingestion & & & \\
\hline
\end{tabular}

Values are shown as means and standard deviation (SD). Significance is shown using an $*(p<0.05$.). Large effect size is shown using * $(\mathrm{d}>0.8)$

Table 4. Circulating $\beta$ hb Concentration. $\beta$ hb Concentration Was Measured Before Ingesting the Supplement and Again 30 Minutes after; During the Post-Ingestion Protocol

\begin{tabular}{|c|c|c|c|c|}
\hline Supplement & Mean $(\mathrm{mmol} / \mathrm{L})$ & SD & P-Value & Cohen's D \\
\hline СHO & & & 0.443 & 0.246 \\
\hline 0 minutes & 0.11 & 0.05 & & \\
\hline 30 minutes & 0.13 & 0.10 & & \\
\hline PLA & & & 0.200 & 0.277 \\
\hline 0 minutes & 0.24 & 0.35 & & \\
\hline $30 \mathrm{~min}$ post & 0.35 & 0.43 & & \\
\hline LKET & & & 0.301 & 0.505 \\
\hline 0 minutes & 0.30 & 0.29 & & \\
\hline 30 min post & 0.53 & 0.63 & & \\
\hline HKET & & & $0.004^{*}$ & $1.892^{*}$ \\
\hline 0 minutes & 0.08 & 0.16 & & \\
\hline 30 min post & 0.36 & 0.13 & & \\
\hline
\end{tabular}

Values are shown in means and standard deviation (SD). Significance is shown using $*(p<0.05$. $)$. A large effect size is shown using $*(\mathbf{d}>0.8)$

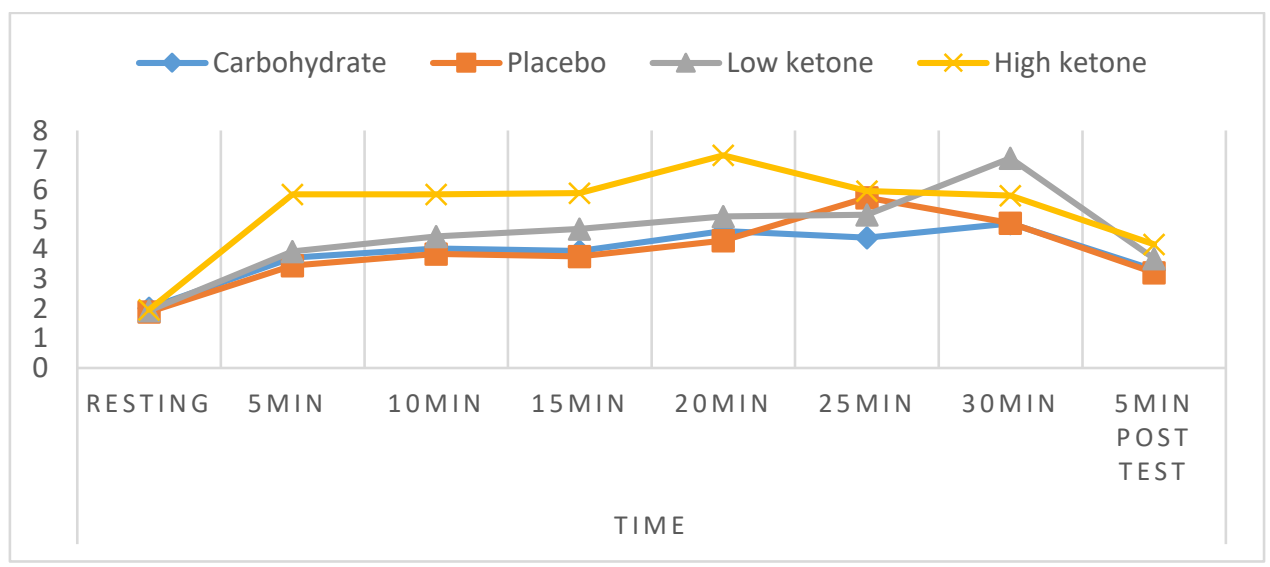

Figure 2. Blood Lactate

The figure shows the post-ingestion blood lactate means for all four supplement groups: measured from rest, at five-minute intervals, until five minutes after ending the test (namely: $5 \mathrm{~min}$ post-test).

Maximum Blood Lactate. Maximum blood lactate values are significantly higher postingestion in the HKET group, $\mathrm{t}(8)=-3.356, \mathrm{P}=$
0.010 , reaching a mean value of $8.45 \pm$ $3.47 \mathrm{mmol} / \mathrm{L}$; in comparison to the $\mathrm{CHO}$ group of only $5.23 \pm 1.66 \mathrm{mmol} / \mathrm{L}$, as seen in Figure 2 .

Lactate Threshold. A significant effect size $(d=0.93)$ in the HKET group was found in comparison to the placebo group $(\mathrm{d}=0.38)$, indicating that HKET resulted in a decrease in lactate threshold. The most significant effect size 
was in the CHO group $(\mathrm{d}=1.538)$, where the lactate threshold was increased.

Blood Glucose. No significant changes in blood glucose were found post-ingestion.

Heart Rate, Rate of Perceived Exertion, and Respiratory Exchange Ratio. No significant changes were found with the HR, RER, and RPE values post-ingestion. The RER post-ingestion values between the supplement groups ranged from $0.72-0.75( \pm 0.04)$.

\section{DISCUSSION}

To our knowledge, this is the first study to compare four supplements: two containing different concentrations of $\beta \mathrm{hb}$, with carbohydrate and placebo supplements. The supplements were manufactured to imitate commercial $\beta \mathrm{hb}$ supplements, matching their common ingredients and quantities $(10 \mathrm{~g} \beta \mathrm{hb})$. The key findings of the study included: increased $\mathrm{VO}_{2}$ and blood lactate, and decreased lactate threshold in the high ketone group (HKET).

When comparing the cycle protocols, the HKET increased oxygen consumption $\left(\mathrm{VO}_{2}\right)$, as did the carbohydrate and placebo supplements. Both the pre- and post-ingestion protocols were performed at the same intensity. Thus the rise in $\mathrm{VO}_{2}$ indicates a decrease in exercise response. LKET was the only supplement that preserved $\mathrm{VO}_{2}$ post-ingestion, suggesting a low dosage ketone supplement may maintain current exercise response. Similar studies showed ketone body supplements increased $\mathrm{VO}_{2}$ during high-intensity all-out cycling (2) and a $31 \mathrm{~km}$ time trial (15). This study also showed an increase in $\mathrm{VO}_{2}$; however, the protocols were performed at a moderate intensity of $60 \%$. If a nutritional supplement resulted in improved exercise response, $\mathrm{VO}_{2}$ should decrease.

Previous studies reported ketone supplements reducing blood lactate among cyclists $(9,15)$. Results showed $\sim 50 \%$ lower lactate readings compared to a carbohydrate supplement, and a $6 \mathrm{mmol} / \mathrm{L}$ decrease compared to a placebo $(9,15)$. This study showed a $2.2 \mathrm{mmol} / \mathrm{L}(\mathrm{d}=0.8)$ increase in maximal blood lactate post-ingestion for the HKET group. A few factors could have elicited such results: both Cox et al. and Lecky et al. (9, 15) used highly trained elite athletes for their testing, indicating an increased capacity in maintaining blood lactate $(19,20)$. Additionally, Cox et al. and Lecky et al. $(9,15)$ used pure ketone esters, not $\beta$ hb salts.
Several participants reached the lactate threshold (> 4.00mol/L) during specific points (minutes: seconds) of testing. The HKET group experienced a decrease in the amount of time taken to reach the lactate threshold. The group reached the threshold of $\sim 3$ minutes $(d=-0.935)$ earlier post-ingestion. It was comparing to the $\mathrm{CHO}$ group that experienced a large increase in time to reach the lactate threshold at $\sim 6.66$ minutes $(\mathrm{d}=1.538)$ later during the post-ingestion protocol.

Commercial $\beta \mathrm{hb}$ supplements claim to increase blood $\beta \mathrm{hb}$ concentrations similar to nutritional ketosis $(\beta \mathrm{hb}>0.5-<4.00 \mathrm{mmol} / \mathrm{L})$. Supporting this, one study showed ketone supplements increased blood $\beta$ hb concentration to $<4.00 \mathrm{mmol} / \mathrm{L}$ following ingestion (9). Conflicting results found only small to moderate increases of $<2.0 \mathrm{mmol} / \mathrm{L}-1.3 \mathrm{mmol} / \mathrm{L}(2,12$, 21). This study manufactured the HKET supplement to mimic commercial $\beta \mathrm{hb}$ supplements, containing $\beta \mathrm{hb}$ bound to sodium, similar to the 2018 study by Evans (21). The HKET supplement $(10 \mathrm{~g}$ of $\beta \mathrm{hb})$ showed the largest effect on blood $\beta \mathrm{hb}$, increasing the concentration to a mean value of $0.367 \mathrm{mmol} / \mathrm{L}$, 30 minutes following ingestion. The differences between studies maybe because of the quality of the ketone salt or ester used: the study that elicited the highest response used an ester (9), and the lowest used a salt (2). Other changes in $\beta \mathrm{hb}$ readings may be because of different pre-testing nutrition strategies (1). This study did not provide participants with pre-testing meals but instead advised participants to avoid eating two hours before testing and for keeping a 24-hour food journal. This action ensured they ate similar meals before the pre- and post-ingestion protocols. This protocol is similar to studies where pre-exercise nutrition was self-regulated (2). Although the LKET supplement resulted in no significant increase from baseline to 30 minutes postingestion, it did result in ketosis $(0.53 \mathrm{mmol} / \mathrm{L} \pm$ $0.63)$. This result may be due to the group having higher baseline $\beta \mathrm{hb}$ concentrations.

None of the participants in the current study reported adverse side effects, despite participants in other studies experiencing gut discomfort (2, $12-15)$. It is possible the dosage of $\beta \mathrm{hb}$ was not high enough $(<10 \mathrm{~g})$ to cause harmful side effects. This study can not determine if repeated use would cause adverse effects as the supplements were only administered once. RER was not 
affected when comparing pre- to post-ingestion that suggests that the $\beta \mathrm{hb}$ did not affect substrate utilization, or the quantity of $10 \mathrm{~g}$ was too small.

This study did have limitations including testing times were not consistent between preand post-ingestion protocols; macronutrients were not standardized in the pre-testing meals due to the self-regulation strategy applied; $\beta \mathrm{hb}$ concentrations were not tested as frequently as blood glucose and blood lactate due to costs restraints; lactate threshold was not tested during the baseline VO2max test in addition to the submaximal cycle protocol, and a cross-over design was not used due to time restraints.

These results can be used to raise awareness on efficacy as well as ingredients and label compliance of ketone supplements. Due to the large number of inconsistencies found between the current study and previous studies, further testing is needed. Testing should include the comparison of ketone salts to esters to determine which form is suited as an ingredient for supplements. Additionally, ketone supplements and dietary intake to instruct consumers on a recommended diet to be followed while taking ketone supplementation. Lastly, testing should be conducted following long-term ingestion to establish long-term effects.

\section{CONCLUSION}

Study results vary in the current literature that resulted in a lack of evidence supporting the quantity and type of ketone bodies used in commercial supplements to improve exercise response. This study's supplements contained exogenous $\beta \mathrm{hb}$ salts. The supplements were able to induce a mild state of ketosis $(>0.5 \mathrm{mmol} / \mathrm{L})$; however, they increased oxygen consumption and blood lactate, showing an acute decrease in exercise response. It has been shown that $10 \mathrm{~g}$ of $\beta$ hb did not benefit exercise response.

\section{APPLICABLE REMARKS}

- Consider the effects of $\beta$ hb supplements.

- Consumer awareness can be improved regarding the efficacy and ingredients in commercial ketone supplements.

- $10 \mathrm{~g} \beta \mathrm{hb}$ showed no benefit to exercise.

- Caution should be taken when ingesting ketone body supplements due to the lack of knowledge on long term effects.

\section{ACKNOWLEDGMENTS}

The authors thank PRIME Human Performance Institute for allowing all testing to be conducted in a controlled clinical environment.

\section{FUNDING}

The research reported in this publication was supported by the Fogarty International Centre (FIC), National Institutes of Health (NIH) Common Fund, Office of Strategic Coordination, Office of the Director (CF/OSC/OD/NIH), Office of AIDS Research, Office of the Director (OAR/OD/NIH), National Institute of Mental Health (NIMH/NIH), award number D43TW010131. The content is solely the responsibility of the authors and does not necessarily represent the official views of the NIH.

\section{DISCLOSURE OF INTEREST}

The authors report no conflict of interest.

\section{REFERENCES}

1. Pinckaers PJ, Churchward-Venne TA, Bailey D, van Loon LJ. Ketone Bodies and Exercise Performance: The Next Magic Bullet or Merely Hype? Sports Med. 2017;47(3):383-391. doi: 10.1007/s40279-016-0577-y pmid: 27430501

2. Rodger S, Plews D, P. L, Driller M. Oral B-hydroxybutyrate salt fails to improve 4-minute cycling performance following submaximal exercise. J Sci Cycl. 2017;6(1):26-31.

3. Harvey C, Schofield GM, Williden M. The use of nutritional supplements to induce ketosis and reduce symptoms associated with keto-induction: a narrative review. PeerJ. 2018;6:e4488. doi: 10.7717/peerj.4488 pmid: 29576959

4. Niknamian S. Nutritional ketosis condition and specific ketogenic diet, may benefit cancer patients as an alternative treatment by sudden change in the metabolic state of cancer cells. Int J Biochem Physiol. 2016;1(1). doi: 10.23880/IJBP-16000107

5. Clarke K, Cox P. Ketone bodies and ketone body esters for maintaining or improving muscle power output. United State Patent Applicat. 2015.

6. Holdsworth DA, Cox PJ, Kirk T, Stradling H, Impey SG, Clarke K. A Ketone Ester Drink Increases Postexercise Muscle Glycogen Synthesis in Humans. Med Sci Sports Exerc. 2017;49(9):1789-1795. doi: 10.1249/MSS.0000000000001292 pmid: 28398950

7. Stubbs BJ, Cox PJ, Evans RD, Santer P, Miller JJ, Faull OK, et al. On the Metabolism of Exogenous Ketones in Humans. Front Physiol. 2017;8:848. doi: 10.3389/fphys.2017.00848 pmid: 29163194 
8. Egan B, D'Agostino DP. Fueling Performance: Ketones Enter the Mix. Cell Metab. 2016;24(3):373-375. doi: 10.1016/j.cmet.2016.08.021 pmid: 27626197

9. Cox PJ, Kirk T, Ashmore T, Willerton K, Evans R, Smith A, et al. Nutritional Ketosis Alters Fuel Preference and Thereby Endurance Performance in Athletes. Cell Metab. 2016;24(2):256-268. doi: 10.1016/j.cmet.2016.07.010 pmid: 27475046

10. Ramos-Jimenez A, Hernandez-Torres RP, Torres-Duran PV, Romero-Gonzalez J, Mascher D, Posadas-Romero C, et al. The Respiratory Exchange Ratio is Associated with Fitness Indicators Both in Trained and Untrained Men: A Possible Application for People with Reduced Exercise Tolerance. Clin Med Circ Respirat Pulm Med. 2008;2:19. doi: $10.4137 /$ ccrpm.s449 pmid: 21157516

11. Cox PJ, Clarke K. Acute nutritional ketosis: implications for exercise performance and metabolism. Extrem Physiol Med. 2014;3:17. doi: 10.1186/2046-7648-3-17 pmid: 25379174

12. Evans M, McSwiney FT, Brady AJ, Egan B. No Benefit of Ingestion of a Ketone Monoester Supplement on 10km Running Performance. Med Sci Sports Exerc. 2019;51(12):2506-2515. doi: 10.1249/MSS.0000000000002065 pmid: 31730565

13. Stubbs BJ, Cox PJ, Kirk T, Evans RD, Clarke K. Gastrointestinal Effects of Exogenous Ketone Drinks are Infrequent, Mild and Vary According to Ketone Compound and Dose. Int J Sport Nutr Exerc Metab. 2019:1-23. doi: 10.1123/ijsnem.2019-0014 pmid: 31034254

14. Vandoorne T, De Smet S, Ramaekers M, Van Thienen R, De Bock K, Clarke K, et al. Intake of a Ketone Ester Drink during Recovery from Exercise Promotes mTORC1 Signaling but Not Glycogen Resynthesis in Human Muscle. Front Physiol. 2017;8:310. doi: 10.3389/fphys.2017.00310 pmid: 28588499

15. Leckey JJ, Ross ML, Quod M, Hawley JA, Burke LM. Ketone Diester Ingestion Impairs Time-Trial Performance in Professional Cyclists. Front Physiol. 2017;8:806. doi: 10.3389/fphys.2017.00806 pmid: 29109686

16. Fischer T, Och U, Klawon I, Och T, Gruneberg M, Fobker M, et al. Effect of a Sodium and Calcium DL-betaHydroxybutyrate Salt in Healthy Adults. J Nutr Metab. 2018;2018:9812806. doi: 10.1155/2018/9812806 pmid: 29850235

17. Hagberg JM, Mullin JP, Giese MD, Spitznagel E. Effect of pedaling rate on submaximal exercise responses of competitive cyclists. J Appl Physiol Respir Environ Exerc Physiol. 1981;51(2):447-451. doi: 10.1152/jappl.1981.51.2.447 pmid: 7263451

18. Lucia A, Hoyos J, Perez M, Santalla A, Chicharro JL. Inverse relationship between VO2max and economy/efficiency in world-class cyclists. Med Sci Sports Exerc. 2002;34(12):2079-2084. doi: 10.1249/01.MSS.0000039306.92778.DF pmid: 12471319

19. Tanner R, Gore C. Physiological tests for elite athletes.2013. 546 p.

20. Czuba M, Zajac A, Cholewa J, Poprzecki S, Waskiewicz Z, Mikotajec K. Lactate Threshold (D-Max Method) and Maximal Lactate Steady State in Cyclists. J Human Kinetic. 2009;21:49-56. doi: 10.2478/v10078-09-0006-5

21. Evans M, Patchett E, Nally R, Kearns R, Larney M, Egan B. Effect of acute ingestion of beta-hydroxybutyrate salts on the response to graded exercise in trained cyclists. Eur J Sport Sci. 2018;18(3):376-386. doi: 10.1080/17461391.2017.1421711 pmid: 29338584 